\title{
BMJ Open Therapeutic effects of exercise interventions for patients with chronic kidney disease: protocol for an overview of systematic reviews and meta-analyses of clinical trials
}

\author{
Fan Zhang (D) , ${ }^{1}$ Hui Wang, ${ }^{1}$ Liuyan Huang, ${ }^{1}$ Huachun Zhang ${ }^{2}$
}

To cite: Zhang $\mathrm{F}$, Wang $\mathrm{H}$, Huang L, et al. Therapeutic effects of exercise interventions for patients with chronic kidney disease: protocol for an overview of systematic reviews and meta-analyses of clinical trials. BMJ Open 2021;11:e043011. doi:10.1136/ bmjopen-2020-043011

- Prepublication history for this paper is available online. To view these files, please visit the journal online (http://dx.doi. org/10.1136/bmjopen-2020043011).

Received 13 August 2020 Revised 18 January 2021 Accepted 21 January 2021

Check for updates

(C) Author(s) (or their employer(s)) 2021. Re-use permitted under CC BY-NC. No commercial re-use. See rights and permissions. Published by BMJ.

${ }^{1}$ Department of Nephrology, Long Hua Hospital, Shanghai, China

${ }^{2}$ Department of Nursing, Long Hua Hospital, Shanghai, China

Correspondence to

Dr Fan Zhang;

1051071914@qq.com

\section{ABSTRACT}

Introduction An increasing number of systematic

reviews and meta-analyses of clinical trials have begun to investigate the effects of exercise interventions in patients with chronic kidney disease (CKD). To systematically appraise and synthesise these results, we will conduct an overview of systematic reviews and meta-analyses.

Methods and analysis This is a protocol for an overview of systematic reviews and meta-analyses. We will search four databases: PubMed, Embase, Web of Science Core Collection and Cochrane Central Registry of Controlled Trials. Systematic reviews and meta-analyses of clinical trials evaluating the effect of exercise interventions on patients with CKD will be included. Two independent authors will screen titles and abstracts retrieved in the literature search and select studies meeting the eligibility criteria for full-text review. The methodological quality of the included reviews will be assessed using $A$ Measurement Tool to Assess Systematic Reviews-2. We estimate the summary effects for each meta-analyses based on the standardised mean difference and its 95\% $C l$. Additionally, the heterogeneity will be assessed by $P$ evidence of small-study effect and the quality of evidence using the Grading of Recommendations Assessment, Development and Evaluation system.

Ethics and dissemination Ethics approval are not required as no private information from individuals is collected. The results will be published in a peer-reviewed journal or disseminated in relevant conferences.

PROSPERO registration number CRD42020223591.

\section{INTRODUCTION}

Chronic kidney disease (CKD) represents an enormous burden of morbidity across the globe affecting about $10.4 \%-16 \%$ of all adults. ${ }^{1-3}$ In recent years, with the rising incidence of hypertension and diabetes mellitus, the number of patients with CKD will continue to grow. ${ }^{45}$ During the progression of CKD, patients can experience a range of physical and psychological side effects associated with their disease or treatment, such as impaired physical function, fatigue, not only
Strengths and limitations of this study

- A strength of this review is that it is the present overview of clinical trials of the effectiveness of exercise interventions on health problems among patients with chronic kidney disease, including dialysis and renal transplant.

- Another strength is that our method allows us to map the available evidence and identify where the evidence base is strong or weak.

- The main limitation of this review is that only articles published in the English language will be included. Another limitation is that only published studies will be eligible for inclusion.

results in a diminished health-related quality of life (HRQOL) but impacts patients' activities in daily life. ${ }^{67}$ Reports of these poor prognoses among patients with CKD have created a greater emphasis on lifestyle interventions that reduce complications and improve HRQOL for them. ${ }^{8-10}$

Exercise is a proven intervention associated with numerous health benefits in patients with CKD, and they are now encouraged to participate in insufficient levels of regular physical activity in daily life. ${ }^{112}$ A growing number of systematic reviews and metaanalyses have examined the role of exercise in CKD treatment. Where multiple metaanalyses have been published on similar and overlapping questions over a relatively short time frame, there is a need for an overview of the meta-analyses data, namely umbrella review, to determine the overarching quality and strength of the evidence and the level of consistency or potential contradiction in outcomes. ${ }^{13}$

Based on these premises and the increasing number of systematic reviews on the efficacy of exercise interventions in CKD patients, we 
performed this umbrella review to highlight the specific goals of exercise in rehabilitation in patients with CKD and examine the therapeutic effects of exercise interventions regarding cardiovascular risk factors, physical fitness, dialysis-related symptoms and HRQOL in patients with CKD. Cardiovascular risk factors and physical fitness were investigated because health outcomes that are known to respond favourably to exercise in healthy populations and non-communicable diseases. ${ }^{1415}$ Dialysisrelated symptoms and HRQOL were investigated because they are often adversely affected by the deterioration of renal function or medication treatment side effects. ${ }^{16-18}$

\section{Objectives}

This overview aims to appraise and synthesise published systematic reviews and meta-analyses investigating the therapeutic effects of exercise interventions compared with routine care on health outcomes in patients with CKD. Our overview will explore the similarities and differences in the full text of systematic reviews and metaanalyses to achieve this objective. The results of our findings will be presented as a descriptively report.

\section{METHODS AND ANALYSIS}

This overview followed the Preferred Reporting Items for Systematic Reviews and Meta-Analyses statement for reporting systematic reviews. ${ }^{19}$

\section{Search strategy}

A systematic search will be conducted using the following database: PubMed, Embase, Web of Science Core Collection and Cochrane Central Registry of Controlled Trials. The retrieval time spans from the construction of the database to December 2020. A manual review of references from primary or similar systematic review articles will also be screened to trace additional relevant studies. The search phrase consisted of medical subject heading (MeSH) terms and keywords related to exercise, meta-analyses and CKD (table 1). Article language was restricted to English.

\section{Eligibility criteria}

The eligibility criteria of review will be described as follows: (1) include patients diagnosed with CKD whether it is dialysis or renal transplant or not; (2) compare exercise interventions no matter types with sham/no exercise or usual/standard care; (3) be systematic review with meta-analyses of controlled parallel intervention trials (eg, systematic reviews with meta-analyses of randomised controlled trials (RCTs) and quasi-experimental studies were eligible, systematic reviews with meta-analyses of prospective observational studies were excluded); (4) systematic reviews that report outcomes on at least one of the following: cardiovascular risk factors, physical fitness, dialysis-related symptoms and HRQOL. The metaanalyses include less than three studies, or less than 100 participants will be excluded.
Among the outcomes, the cardiovascular risk factor is based mainly on blood pressure and another specific cardiac function indicator like the left ventricular mass index. Physical fitness, including cardiopulmonary fitness, muscle strength, muscle endurance, body composition and flexibility. Dialysis-related symptoms are based principally on the three symptoms: restless legs syndrome, fatigue, dialysis adequacy. HRQOL is measured by any quality of life scales, such as the Short-Form Health Survey.

\section{Study records}

\section{Selection process}

Two independent reviewers will screen titles and abstracts for potential relevance first, then retrieve the potential full-text articles and assess against the eligibility criteria by another two reviewers. In case of disagreement, consensus will be resolved through discussion to reach a consensus or involve a third reviewer.

\section{Data items}

Data will be extracted from each included meta-analyses using a predefined form, included: (1)authors; (2) publication year; (3) stage of CKD, for example, predialysis, peritoneal dialysis, haemodialysis or kidney transplant; (4) if the subjects were haemodialysis patients, data will add an item, namely, exercise performed during dialysis session or non-dialysis day; (5) description of outcome measures; (6) number of included studies and participants; (7) results of risk of bias assessment; (8) number of exercise-related adverse events; (9) study-specific standardised mean difference (SMD) with corresponding $95 \%$ CI.

\section{Quality appraisal of included reviews}

The methodological quality of each included systematic review will be evaluated using the 16 domains described in A Measurement Tool to Assess Systematic Reviews-2 (AMSTAR-2) tool. ${ }^{20}$ AMSTAR-2 is a comprehensive tool aimed to assess the methodological quality of systematic reviews. Any differences between author assessments will be resolved by discussion or adjudication by a third reviewer.

Additionally, the Grading of Recommendations Assessment, Development and Evaluation (GRADE) system will evaluate the quality of evidence provided by each metaanalyses. ${ }^{21}$ See table 2 for a further description of the GRADE system.

\section{Data analysis}

The meta-analyses will be classified according to exercise types into four categories: (1) aerobic exercise; (2) resistance training; (3) aerobic and resistance training combined; (4) other exercise-based therapy. For each category, the summary effect size from each meta-analyses will be analysed qualitatively based on the SMD and its $95 \%$ CI. If there were not presented as SMD in the original meta-analyses, the software of Review Manager V.5.3 will be used to convert the outcomes to SMD to allow visual comparison of the results in a forest plot. If data are insufficient for conversion, which 


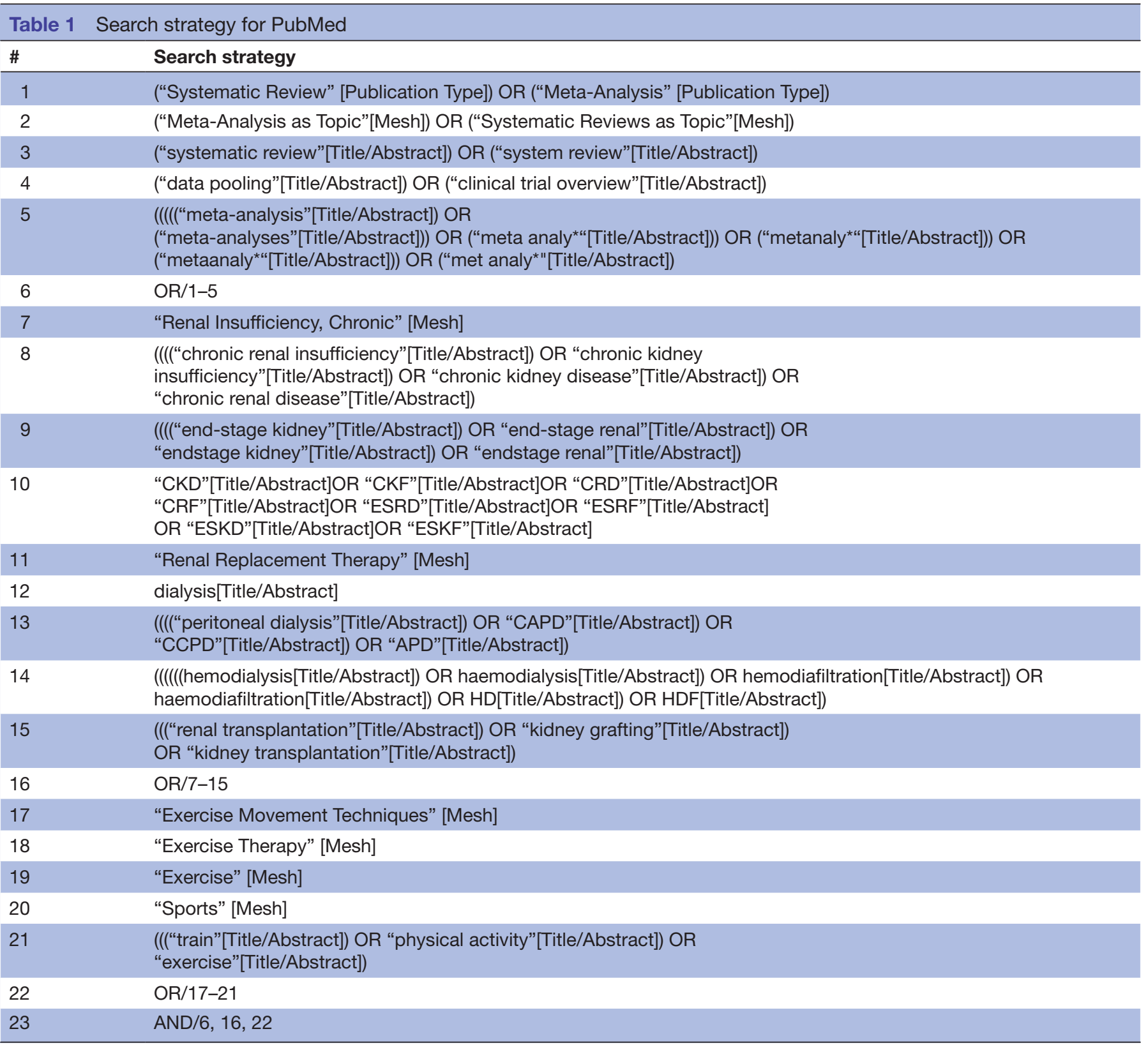

will be extracted from the original RCTs and processed as instructed by the Cochrane Collaboration. The summary effects are considered small $(<0.5)$, moderate $(0.50-0.79)$ and large $(\geq 0.80) .^{22}$

Evidence of small-study effects will be assessed with Egger's regression asymmetry test, and $p<0.10$ with more conservative effect in larger studies than in random-effects meta-analyses will be judged be evidence for small-study effects. ${ }^{23}$

We will also obtain the $I^{2}$ statistic to determine heterogeneity: less than $25 \%$ indicates no heterogeneity; 25\%-49\% indicates low heterogeneity; $50 \%-74 \%$ indicates moderate heterogeneity and $75 \%$ or more indicates high heterogeneity. ${ }^{24}$

Furthermore, we will perform a sensitivity analysis to explore if outcomes change when removing the above studies. Finally, a funnel plot will be performed in order to assess evidence for publication bias and if the reported effect is valid.

Patient and public involvement

Patients and public will not be involved.

\section{DISCUSSION}

Patients with CKD suffer a high burden of high cardiovascular mortality and muscle impairment, contributing to functional decline and mobility disability. ${ }^{25}$ The case for targeting exercise as an intervention in patients with CKD, whether they receive dialysis or renal transplant or not, is vital. ${ }^{11}$ Published reviews on the effects of exercise have reflected this, where variations across the systematic reviews in the interventions considered make it difficult for healthcare providers and policy-makers to identify the 
Table 2 GRADE assessment

\section{Grade recommendation}

High quality

Interpretation

High quality

We are very confident that the true effect lies close to that of the estimate of the effect.

Moderate quality

We are moderately confident in the effect estimate; the true effect is likely to be close to the estimate of effect, nevertheless, there is a possibility that it is substantially different.

$\begin{array}{ll}\text { Low quality } & \begin{array}{l}\text { Our confidence in the effect } \\ \text { estimate is limited; the true effect } \\ \text { may be substantially different from } \\ \text { the estimate of the effect. }\end{array} \\ \text { We have very little confidence in } \\ \text { the effect estimate; the real effect } \\ \text { is likely to be substantially different } \\ \text { from the estimate of effect. }\end{array}$

components of exercise prescription that are successful and affect positive change or replicate these clinically.

Overview of systematic reviews and meta-analyses as a methodological approach is a relatively new and developing field. Our overview will identify the currently available evidence by the systematic reviews that support the published best practice recommendations on exercise interventions for patients with CKD, aim to identify knowledge gaps in our understanding of exercise and prognosis among patients with $\mathrm{CKD}$, and hope to make recommendations for future research based on the results.

Few potential limitations should be considered. First, only articles published in English will be included, leading to evidence selection bias as studies published in other languages will be excluded. Second, only published studies will be eligible for inclusion. While we recognise this could introduce reporting bias into our results, this will be necessary to ensure enough information is available for data extraction and quality appraisal. Third, there may be several relevant studies included, which can cause difficulties in summarising and interpreting results.

Contributors $\mathrm{FZ}$ and HW designed and drafted the protocol. LH and $\mathrm{HZ}$ advised and participated in designing the overview of systematic reviews and contributed to the manuscript. All authors read, provided feedback and approved the final manuscript.

Funding The authors have not declared a specific grant for this research from any funding agency in the public, commercial or not-for-profit sectors.

Competing interests None declared.

Patient and public involvement Patients and/or the public were not involved in the design, or conduct, or reporting, or dissemination plans of this research.

Patient consent for publication Not required.

Provenance and peer review Not commissioned; externally peer reviewed.

Open access This is an open access article distributed in accordance with the Creative Commons Attribution Non Commercial (CC BY-NC 4.0) license, which permits others to distribute, remix, adapt, build upon this work non-commercially, and license their derivative works on different terms, provided the original work is properly cited, appropriate credit is given, any changes made indicated, and the use is non-commercial. See: http://creativecommons.org/licenses/by-nc/4.0/.
ORCID iD

Fan Zhang http://orcid.org/0000-0003-4692-0526

\section{REFERENCES}

1 Mills $\mathrm{KT}, \mathrm{Xu} \mathrm{Y}$, Zhang W, et al. A systematic analysis of worldwide population-based data on the global burden of chronic kidney disease in 2010. Kidney Int 2015;88:950-7.

2 Ricardo AC, Flessner MF, Eckfeldt JH, et al. Prevalence and correlates of CKD in Hispanics/Latinos in the United States. Clin J Am Soc Nephrol 2015;10:1757-66.

3 Zhang L, Wang F, Wang L, et al. Prevalence of chronic kidney disease in China: a cross-sectional survey. Lancet 2012;379:815-22.

4 Zhang L, Long J, Jiang W, et al. Trends in chronic kidney disease in China. N Engl J Med 2016;375:905-6.

5 Yang $\mathrm{C}$, Wang $\mathrm{H}$, Zhao X, et al. Ckd in China: evolving spectrum and public health implications. Am J Kidney Dis 2020;76:258-64.

6 Janmaat CJ, van Diepen M, Meuleman Y. Kidney function and symptom development over time in elderly patients with advanced chronic kidney disease: results of the equal cohort study. Nephrol Dial Transplant 2020:gfz277.

7 van de Luijtgaarden MWM, Caskey FJ, Wanner C, et al. Uraemic symptom burden and clinical condition in women and men of $\geq 65$ years of age with advanced chronic kidney disease: results from the EQUAL study. Nephrol Dial Transplant 2019;34:1189-96.

8 Hendriks FK, Smeets JSJ, van der Sande FM, et al. Dietary protein and physical activity interventions to support muscle maintenance in end-stage renal disease patients on hemodialysis. Nutrients 2019;11:2972.

9 Sheshadri A, Kittiskulnam P, Lazar AA, et al. A walking intervention to increase Weekly steps in dialysis patients: a pilot randomized controlled trial. Am J Kidney Dis 2020;75:488-96.

10 Evangelidis N, Craig J, Bauman A, et al. Lifestyle behaviour change for preventing the progression of chronic kidney disease: a systematic review. BMJ Open 2019;9:e031625.

11 Zelle DM, Klaassen G, van Adrichem E, et al. Physical inactivity: a risk factor and target for intervention in renal care. Nat Rev Nephrol 2017;13:152-68.

12 Mallamaci F, Pisano A, Tripepi G. Physical activity in chronic kidney disease and the exercise introduction to enhance trial. Nephrol Dial Transplant 2020;35:ii18-22.

13 Aromataris E, Fernandez R, Godfrey CM, et al. Summarizing systematic reviews: methodological development, conduct and reporting of an umbrella review approach. Int J Evid Based Healthc 2015;13:132-40.

$14 \mathrm{Li} \mathrm{G}$, Li J, Gao F. Exercise and cardiovascular protection. Adv Exp Med Biol 2020;1228:205-16.

15 Gronek P, Wielinski D, Cyganski P, et al. A review of exercise as medicine in cardiovascular disease: pathology and mechanism. Aging Dis 2020;11:327-40.

16 Zhou W, Hu W, Han G, et al. The impact of residual renal function on quality of life in patients with peritoneal dialysis. Clin Nephrol 2018;90:106-11.

17 Chin HJ, Song YR, Lee JJ, et al. Moderately decreased renal function negatively affects the health-related quality of life among the elderly Korean population: a population-based study. Nephrol Dial Transplant 2008;23:2810-7.

18 Termorshuizen F, Korevaar JC, Dekker FW, et al. The relative importance of residual renal function compared with peritoneal clearance for patient survival and quality of life: an analysis of the Netherlands Cooperative Study on the Adequacy of Dialysis (NECOSAD)-2. Am J Kidney Dis 2003;41:1293-302.

19 Moher D, Shamseer L, Clarke M, et al. Preferred reporting items for systematic review and meta-analysis protocols (PRISMA-P) 2015 statement. Syst Rev 2015;4:1.

20 Shea BJ, Reeves BC, Wells G, et al. AMSTAR 2: a critical appraisal tool for systematic reviews that include randomised or non-randomised studies of healthcare interventions, or both. BMJ 2017;358:j4008.

21 Atkins D, Best D, Briss PA, et al. Grading quality of evidence and strength of recommendations. BMJ 2004;328:1490.

22 Cohen J. Quantitative methods in psychology: a power primer. Psychol Bull 1992;112:115-59.

23 Belbasis L, Bellou V, Evangelou E, et al. Environmental risk factors and multiple sclerosis: an umbrella review of systematic reviews and meta-analyses. Lancet Neurol 2015;14:263-73.

24 Higgins JPT, Thompson SG, Deeks JJ, et al. Measuring inconsistency in meta-analyses. BMJ 2003;327:557-60.

25 Roshanravan B, Gamboa J, Wilund K. Exercise and CKD: skeletal muscle dysfunction and practical application of exercise to prevent and treat physical impairments in CKD. Am J Kidney Dis 2017;69:837-52. 